El marketing como factor fundamental del desarrollo de la innovación empresarial, caso práctico en Barcelona Activa.

Autor: Jhonatan Steven Lizarazo Guerrero.

C.C 1023926445

Condigo: 2112227

Facultad: Administración de Empresas.

Universidad Santo Tomas

2015 


\section{El marketing como factor fundamental del desarrollo de la innovación empresarial, caso práctico en Barcelona Activa.}

\section{Introducción-Antecedentes:}

Con la evolución de las tecnologías y el actual mundo globalizado las nuevas ideas de negocio son una realidad que observamos a diario; la innovación se refleja en todos los ámbitos de la sociedad y ha permitido satisfacer necesidades que muchos no sabían que se podían satisfacer consolidándose así como un factor determinante en los planes de cualquier gerente; en términos generales: "Una innovación es la puesta en marcha de un producto, un proceso, un método de marketing, o una organización interna, nuevo, o mejorado de forma significativa" (OCDE, 1997 ) 1; y específicamente según Peter Drucker: "innovar es crear una nueva forma de satisfacer una necesidad, aportando más valor a los usuarios. Innovar implica generar novedades que supongan mejoras importantes para los usuarios" (Drucker, 1954) 2.

Este primer escenario nos demuestra la importancia de la innovación empresarial, al respecto se puede agregar que "Compañías de gran éxito como Toys "R" Us, Inditex, Coca-Cola, IKEA o Quicksilver relacionan sus resultados con la aplicación de los principios de la innovación estratégica y reiteradamente en todos los ámbitos de la organización." 3 (Moreno, 2007)

Otro escenario nos muestra al marketing, esta es un área que se ha constituido como primordial para la promoción y consolidación en el mercado de cualquier tipo de producto o servicio; según la American Marketing Asociation, "el marketing es una función de la organización y un conjunto de procesos para crear, comunicar y entregar valor a los clientes, y para manejar las relaciones con estos últimos, de manera que beneficien a toda la organización..." 4 ((A.M.A.)). Por otro lado un experto como Philip Kotler propone: "el marketing es un proceso social $y$ administrativo mediante el cual grupos e individuos obtienen lo que necesitan y desean a través de generar, ofrecer e intercambiar productos de valor con sus semejantes" 5 (Kotler, 2001)

Teniendo en cuenta lo anterior resulta visible la importancia del marketing en el siglo XXI, miles de productos y servicios no han podido trascender ni ser competitivos en el mercado debido a que no tenían un correcto plan de mercadeo, esto a pesar de tener buenas características y ser funcionales en sus áreas; al respecto se puede comentar: "Una cosa son las buenas ideas y otra muy diferente es desarrollar un producto y lanzarlo con éxito y con la aceptación de los consumidores" 6 (Curewitz, Marekting e innovación el consumidor en el punto de mira.)

Después de hacer una breve descripción de la situación de los dos pilares de este trabajo: el marketing y la innovación, se puede formular la hipótesis que servirá como eje de toda la investigación: ¿es realmente el marketing un factor determinante en un proceso de innovación empresarial? 
Con el fin de esclarecer este interrogante en primer lugar se analizaran 5 artículos de autores con autoridad en el tema y se aplicara lo extraído de estos para la resolución de esta pregunta problema; adicionalmente se tendrá en cuenta dos listas de chequeo en referencia a las temáticas, estas fueron aplicadas en base la visita a Barcelona Activa, acerca de esta organización se puede mencionar que: "es la organización ejecutora de las políticas de promoción económica del Ayuntamiento de Barcelona. Desde hace más de 25 años impulsa el crecimiento económico de Barcelona y su ámbito de influencia apoyando a las empresas, a la iniciativa emprendedora y el empleo."7(Barcelona Activa)

De esta visita se obtuvieron valiosos aportes en referencia a estas temáticas ya que de forma más práctica se puede soportar o desmentir la hipótesis planteada, esta empresa es una autoridad en el tema de innovación y emprendimiento empresarial, al respecto algunas cifras: "Barcelona Activa ha atendido 4.293 empresas mediante sus servicios y programas de apoyo empresarial, que se integrarán en la Oficina de Atención a la Empresa y ha acompañado 2.653 proyectos emprendedores. Además, 132 empresas y 15 proyectos se han instalado en sus incubadoras. En el ámbito de Empleo, se han atendido 19.332 personas, un $46 \%$ de las cuales han encontrado trabajo en un periodo de seis meses"8 (Barcelona Activa)

Con estos elementos: primero el marco teórico propuesto y segundo la aplicación práctica de la visita empresarial (listas de chequeo) se extraerán unos resultados alcanzados que permitirán sacar las conclusiones pertinentes respecto a este tema.

\section{Objetivos:}

*General: Determinar el nivel de importancia que tiene el marketing como factor impulsador de la innovación empresarial.

*Específicos: Observar en la empresa visitada (Barcelona Activa) como el marketing se relaciona con la innovación empresarial y establecer estrategias que puedan ser adoptadas en el mercado colombiano.

\section{Justificación:}

Tanto el marketing como la innovación se han consolidado como temas de interés prioritario en la agenda de cualquier gerente, en el actual mundo competitivo innovar es cada vez más complicado en un mercado donde ya todo se ha hecho y donde consolidar una buena idea requiere de un gran ingenio o en determinados casos de un amplio capital; pero ¿porque en ocasiones estas dos prioridades se estudian desde ópticas diferentes? La innovación es vista normalmente como un factor de emprendimiento y se relaciona con áreas como la planeación estratégica; por otro lado el marketing se estudia aparte y se relaciona con áreas como la 
publicidad y la logística; ante esto el autor chileno Alejandro Schnarch Kirberg, menciona "Creatividad, innovación y marketing son conceptos normalmente analizados por separado; sin embargo, no sólo están integrados sino que se constituyen en los factores críticos del éxito en la creación, desarrollo y lanzamiento de nuevos productos y servicios al mercado, ya sea en una empresa establecida o creando una nueva para tal efecto."9 (KIRBERG, Creatividad Aplicada, 2008)

Si tenemos en cuenta esto parece evidente que para que una innovación sea exitosa es necesario que se realice un adecuado estudio de mercadeo, en el cual se estudien las necesidades de determinado segmento del mercado y en el cual se determine la factibilidad de que nuestro producto o servicio sea competitivo; "Ahora más que nunca, la innovación es una clave competitiva fundamental para que las empresas puedan mantener o mejorar su posición en el mercado. De este modo, para mejorar las tasas de éxito de nuevos productos, aumentar la confianza del cliente y afianzar su fidelización es necesario aplicar un modelo de innovación dirigido a ofrecer una óptica respuesta a las necesidades reales de los consumidores."10 (Curewitz, Marekting e innovación el consumidor en el punto de mira.)

Además hay que mencionar que en la actualidad se ven ejemplos de innovaciones que cambian el mercadeo tradicional y se adaptan a los nuevos medios digitales esto demuestra que el marketing también innova a diario "Innovación, Internet, blogs, marketing viral, bulos en la red, suspense, juegos, implicación de los clientes, usuarios convertidos en comerciales y publicistas, opiniones de clientes... Son las señas de identidad de la empresa del siglo XXl"11 (Perez, 2009)

Pero ya sea con marketing tradicional o nuevas formas de marketing como el branding "El Branding ha supuesto un cambio de paradigma fundamental en el marketing de las últimas décadas. El marketing tradicional, centrado en el producto y en sus beneficios, ha dado paso al branding, que pone su énfasis en los significados, en las experiencias y en las relaciones que a partir de ellas se generan"12. El marketing sigue posicionado como una vital herramienta de difusión e innovación, Kirberg menciona al respecto: " $A$ veces se tiene buenas ideas, pero se carece de la claridad para transformarlas en negocios, productos 0 servicios reales, necesarios, deseados y rentables; el marketing es necesario para que esas ideas se trasformen, ya sea en empresas establecidas o nuevas, en innovaciones aceptadas por el mercado"12 (KIRBERG, Marketing Estratégico para Pymes , 2007)

Por ultimo para reafirmar esta relación inexpugnable entre el marketing y la innovación la OCDE los relaciona en el Manual de Oslo de la siguiente manera: "Una innovación en marketing es la puesta en marcha de nuevas técnicas, métodos o planes, que impliquen nuevas o mejoras significativas de: estrategias, posicionamiento o segmentación; de comercialización, distribución o venta; de comunicación, promoción o publicidad; de políticas de fijación de precio; o de diseño de productos o de envases"13 (OCDE, 1997 ) 
Teniendo en cuenta lo anterior parece acertado pensar en el marketing como un factor fundamental del desarrollo de la innovación empresarial; sin embargo solo con un marco referencial apropiado como lo son los artículos expuestos en este trabajo sumado con una experiencia práctica como fue la visita a la empresa Barcelona Activa se podrá corroborar la veracidad de este planteamiento.

\section{Revisión de la literatura:}

\section{1) Titulo: Marketing e Innovación: Los ingredientes perfectos para cocinar un negocio exitoso.}

Autor: Andrés Toledo Mater La Salle - Universidad Ramón Llull

Fuente: Revista puro marketing

"Si en nuestro negocio hemos olvidado que innovar es importante, el fracaso tratará de recordárnoslo"

Seguramente muchos estén familiarizados con este término común. Proveniente del latín "innovare", la innovación según el Diccionario de la Real Academia Española, hace referencia a la "creación o modificación de un producto, y su introducción en un mercado". Su significado y uso más coloquial y extendido es aplicado a todo aquello que se muestra o percibe de forma novedosa, o para referirse a determinadas tendencias y nuevas ideas.

La innovación es sin duda un concepto vinculado y ligado de forma innata al mundo de los negocios, pero a su vez, es un aspecto reservado solo para aquellas empresas visionarias capaces de mantener un pulso continuado con su evolución en el tiempo. Las premisas de la innovación son aquellas que de forma autoexigente, nos hacen plantearnos retos y objetivos de forma permanente para mejorar y ser mucho más competitivos. Y ya lo decía bastante claro Peter Drucker al afirmar que en una empresa, "sólo existen dos funciones básicas, el Marketing y la Innovación".

Podemos comprobar como en cualquier mercado o sector profesional, el nivel de competitividad y la exigencia a las que las empresas son sometidas se mantienen en una especie de tensión constante. Y para diferenciarse o lograr mantenerse en la cumbre, no podemos renegar de las bondades que la innovación y el marketing nos ofrecen para impulsar nuestro negocio con un nuevo plus de valor añadido.

Es por ello, que tanto el Marketing como la Innovación, pueden ser considerados como los ingredientes perfectos de una receta para "cocinar" un negocio exitoso. Sin embargo, aunque muchos negocios recurren al marketing para tal vez, moldear y hacer percibir una falsa imagen distante de la realidad, otras hacen uso de la innovación para convertirse en empresas visionarias y pioneras a las que muchos otros intentan imitar. 
Empresas como Apple, Facebook, Amazon, Google, Walmart o Intel se encuentras reconocidas como algunas de las grandes compañías más innovadoras a nivel mundial. $Y$ como podemos apreciar, el componente tecnológico ante su evolución constante, hace mucho más factible que se produzcan muchas más innovaciones en este terreno. Sin embargo, la innovación no queda reservada solo para grandes corporaciones y empresas. A cualquier nivel profesional las puertas de la innovación siempre quedan abiertas. Sin embargo, "la innovación no está concebida para las personas conformistas. Queda reservada para quienes se muestran dispuestos a arriesgar y emprender a través de las ideas y los grandes cambios"

No hay excusa alguna para no innovar en nuestro negocio, y si cientos de razones para estar dispuesto a ello. "La innovación es el único recurso con el que podemos conseguir diferenciarnos o conseguir que nuestra competencia parezca completamente obsoleta". Ignorar esta realidad y hacer lo contrario conlleva el ceder poco a poco parte de nuestra posición y el terreno ganado en el mercado.

Las empresas con carácter innovador tendrán muchas más posibilidades de ser las empresas líderes del mañana. Y no viene mal el recordar que "el tiempo es el peor enemigo para los que nunca innovan" y que "si en nuestro negocio hemos olvidado que innovar es importante, el fracaso tratará de recordárnoslo". 14 (toledo)

\section{2) Titulo: Innovación en Marketing.}

Autor: Fernando Rivero-CEO de ditrendia, Director del Observatorio de la Blogosfera de Marketing y Miembro del Comité de Dirección de la Asociación de Marketing de España.

Fuente: MK Marketing + Ventas

¿Qué es la innovación en marketing?

La finalidad de las innovaciones en marketing es afrontar mejor las necesidades de los clientes, abrir nuevos mercados, introducir un nuevo producto en el mercado, etc., con el objetivo de incrementar las ventas de nuestra organización.

La diferencia entre una innovación en marketing con otras actividades de marketing llevadas a cabo en nuestra organización es que la primera implica la puesta en marcha de métodos no utilizados previamente.

Debe ser parte de un nuevo concepto de marketing o de una nueva estrategia que suponga un salto cualitativo respecto a los métodos de marketing que la empresa ya esté empleando. Como hemos comentado, este nuevo concepto de marketing puede ser desarrollado internamente, adaptado o "copiado" de otras organizaciones o contratado a empresas externas. En cualquier caso, los nuevos métodos de marketing se pueden poner en marcha tanto para nuevos productos, como para los ya existentes. 
*Innovación en marketing basada en el packaging

Dentro de la innovación en marketing incluimos nuevos o cambios significativos en el diseño o envase (packaging, en inglés) de los productos que formen parte de un nuevo enfoque de marketing. Consideramos cambios en el diseño de productos a los producidos en la forma del producto y en su apariencia que no alteren sus características o su funcionalidad última (ya que en ese caso lo consideraríamos innovación en producto). También contemplamos los cambios en los envases de, por ejemplo, productos alimenticios, bebidas o detergentes, donde el envoltorio es el factor clave para determinar la apariencia del producto.

*Innovación en marketing basada en la comercialización

Innovaciones en marketing basados en la comercialización de productos 0 servicios, ya sean nuevos o no, normalmente conllevan incluir nuevos canales de venta o mejorar significativamente los existentes. Entendemos por canales de venta al método que se sigue para vender nuestros productos a los clientes, y no al aspecto de distribución o logístico (el transporte, el almacenaje o el manipulado del producto), que tendría más que ver con la innovación en procesos.

*Innovación en marketing basada en la comunicación

Nuevos métodos de marketing basados en la comunicación incluyen nuevas formas de dar a conocer los productos o servicios de nuestra organización, tanto en medios como en técnicas de comunicación.

Por ejemplo, el uso por primera vez de famosos en nuestra publicidad, o el incluir nuestros productos en un programa de televisión (product placement, en inglés), se podrían considerar innovaciones en marketing.

El uso de famosos en nuestra publicidad por primera vez es un ejemplo de innovación en marketing basado en la comunicación, como fue en su momento este de Apple: El cambio o desarrollo de un nuevo logotipo, imagen corporativa o imagen de marca (branding, en inglés), que se diferencie de una simple mejora o actualización de nuestro logo, con la finalidad de mejorar o posicionar nuestra empresa en un nuevo mercado, o mejorar la imagen de nuestro producto, también podríamos considerarlo innovación en marketing.

\section{*Innovación en marketing basada en el precio}

Innovaciones en precio implican el uso de estrategias de fijación de precios, nuevas o mejoradas significativamente, para nuestros productos o servicios.

Algunos ejemplos son el uso por primera vez de nuevos métodos para variar el precio de un producto o servicio basado en la demanda que tiene (si hay poca demanda, se bajan los precios).

Sin embargo los métodos de precios cuya finalidad es diferenciar los precios en función del segmento del cliente no los podemos considerar innovaciones de marketing (esto sería simplemente una política de fijación de precios), salvo que sea algo que se haga por primera vez en nuestra organización. 15 (Rivero, 2007) 


\section{3) Titulo: Creatividad, innovación y marketing}

Autor: Alejandro Schnarch Kirberg- catedrático en varias universidades Latinoamericanas y autor de reconocidos libros.

Fuente: Avalon U Tadeo: Con extractos de los libros: Desarrollo de Nuevos Productos y Empresas (McGraw-Hill, 2008); Marketing Estratégico para Pymes (Global Educación, 2007) Y Creatividad Aplicada (Ecoe Ediciones, 2008).

Creatividad, innovación y marketing son conceptos normalmente analizados por separado; sin embargo, no sólo están integrados sino que se constituyen en los factores críticos del éxito en la creación, desarrollo y lanzamiento de nuevos productos y servicios al mercado, ya sea en una empresa establecida o creando una nueva para tal efecto.

El crear empresas o introducir productos inéditos al mercado, trae consigo un riesgo ineludible, tanto en el ámbito tecnológico, como financiero y de mercado, siendo notoria la alta tasa de fracasos, fundamentalmente producida por un manejo y desarrollo muy intuitivo y poco científico, por parte de muchas organizaciones y emprendedores.

Para mejorar esta situación, las actividades involucradas en la creación de empresas y la introducción de nuevos productos, deberían manejarse con criterios y metodologías que han probado disminuir los peligros de un fracaso y aseguren, en alguna medida, el aumentar las posibilidades de éxito.

En las nuevas condiciones competitivas, de globalización e incertidumbre, las tres piedras angulares del emprendimiento son definitivamente la creatividad, la innovación y el marketing. La creatividad se relaciona con la búsqueda y aprovechamiento de oportunidades de ideas que satisfagan necesidades reales y sentidas del mercado; la innovación se refiere a la aplicación y desarrollo de productos y empresas y el marketing no solo ayuda a la identificación de esos requerimientos, sino que aporta al diseño, comercialización e introducción de esas innovaciones a la validación y comercialización.

A veces se tiene buenas ideas, pero se carece de la claridad para transformarlas en negocios, productos o servicios reales, necesarios, deseados y rentables. El libro tiene, precisamente esa finalidad: proporcionar las orientaciones para que esas ideas se trasformen, ya sea en empresas establecidas o nuevas, en innovaciones aceptadas por el mercado

Como dice el autor, "Creatividad sin innovación, es como un sueño; Innovación sin creatividad, no puede existir; Innovación sin marketing no tiene sentido.

Creatividad, innovación y marketing en acción, son las claves del emprendimiento exitoso" 
Muchas veces se piensa que la creatividad es patrimonio de las comunicaciones en general o la publicidad en particular, sin embargo es algo que puede y debe ser aplicado en todas las actividades, tareas y funciones da la administración y el marketing. Por ejemplo, incrementar el servicio ofrecido a los clientes, lanzar nuevos productos, mejorar sistemas o procesos de trabajo, buscar formas de incrementar las ventas, hacer cambios en la logística, etc.

La empresa que no sea capaz de cambiar, de modificarse a sí misma para adaptarse a las nuevas circunstancias presentes y futuras, corre el riesgo de estancarse o desaparecer. Para evitarlo y por el contrario, crecer, se requiere de la capacidad de generar cosas diferentes y originales, es decir de la creatividad, no solo para solucionar problemas o aspectos que afecten negativamente la compañía, sino para indagar sobre nuevos enfoques de gestión que permitan buscar, construir o aprovechar oportunidades para sobrevivir y progresar.

Decíamos que creatividad sin innovación es como un sueño y que innovación sin creatividad no puede existir, pero innovación sin marketing, no tiene sentido. Es justamente el marketing lo que nos permite llevar a la práctica de manera exitosa las ideas, plasmadas en los proyectos empresariales, los nuevos productos 0 servicios y el desarrollo de estrategias acertadas

Pero generalmente los conceptos de creatividad, innovación y marketing se tratan y estudian por separado, sin embargo, como hemos visto, no sólo están relacionados, sino pueden constituir las claves del éxito. 16 (Kirberg)

\section{4) Titulo: Innovación en marketing}

Autor: Santiago Monfort Sabater- Director de PAUTA - Consultores de Organización y RRHH; T (Sabater)he Trebol Company; Coordinador de formación de 'El Periódico del Azulejo'

Fuente: www.clubdemarketing.com

La innovación y el marketing, se están revelando como dos estrategias fundamentales para la competitividad de nuestras empresas. En artículos anteriores, ya hemos visto la conveniencia de la estrategia del marketing, pero ahora toca reflexionar sobre ¿qué es la innovación en marketing?.

En la actual situación del mercado, maduro, global y con exceso de oferta, se necesitan nuevos argumentos para diferenciarnos de la competencia. Las estrategias de sacar novedades, con prestaciones técnicas para diversos usos, con diseños que 'están a la moda', incluso personalizados, no son suficientes.

Resulta más necesario que nunca retener y fidelizar a los clientes porque se nos agota el número de nuevos mercados en los que entrar y el coste de captación de clientes nuevos es muy superior al de mantenerlos. Además, el precio no garantiza la fidelidad de los clientes.

Lo que define el valor de una empresa es su posición en el mercado. Por tanto las estrategias principales a medio y largo plazo que debemos acometer, son 
aumentar las actividades de alto valor ligadas a la producción de alta calidad, la investigación, el diseño, la innovación, la logística, la imagen de marca y la distribución.

Todas estas estrategias pasan por la diferenciación. Una diferenciación que aporte un mayor valor a los clientes, para que estén dispuestos a pagar unos precios superiores, y sobre todo para que no nos abandonen a las primeras de cambio.

Marketing e Innovación:

Gran parte de la innovación en el sector azulejero, viene de la mano de innovaciones de otros, por mejoras en los procesos, que aumentan la productividad y reducen costes, debidas a mejoras que ofrecen los fabricantes de maquinaria y esmaltes. Pero, salvo pocas empresas, la mayoría no están creando ninguna forma nueva de satisfacer las necesidades de los usuarios que les proporcione un valor superior. El principal freno a esta falta de innovación es el éxito en el negocio tradicional, la forma exitosa de hacer las cosas hasta el momento. Esto suele asfixiar el espíritu emprendedor que hay dentro de la empresa.

Las innovaciones que tienen sentido, son válidas y se pueden considerar como tales, son cuando han sido refrendadas con el éxito por el mercado, cuando aportan valor al consumidor. Por eso las empresas innovadoras se centran y enfocan hacia el mercado. Y por tanto, conocen, utilizan y desarrollan el marketing.

En este paradigma, la innovación en marketing supone encontrar ventajas competitivas, para crear nuevos mercados o categorías, o nuevas utilidades y usos dentro de los mercados actuales, o mejorar la posición en el mercado gracias a una nueva forma de gestión y relación con el cliente (final, distribuidor ó prescriptor).

Debemos incorporar estrategias centradas en el cliente y en la innovación continua, tanto en productos como en servicios. El sector debe mejorar sus procesos de comercialización y distribución, debe cualificar a sus técnicos de ventas, y debe fidelizar y colaborar mejor con sus redes de distribución y prescripción, así como mejorar la comercialización en el exterior.

Es necesario explorar formas alternativas de gestionar nuestras empresas. Debemos replantearnos el negocio, y es el momento de pensar en nuevas estrategias empresariales que estén basadas en incrementar el posicionamiento en la cadena de valor hacia el cliente de nuestras empresas. Debemos explorar las ventajas y oportunidades de una nueva gestión empresarial y aumentar el valor de los servicios, que son los puntos más débiles en nuestras empresas.17 (Sabater)

\section{5) Marketing e innovación: el consumidor en el punto de mira}

Autor: Barry Curewitz

Fuente: Revista Harvard-Deusto Marketing \& Ventas, 
Los gestores de marcas se enfrentan siempre a la necesidad de encontrar un equilibrio entre la innovación en nuevos productos, por una parte, y los recursos disponibles y las expectativas de la dirección, por otra. El orden de estas prioridades de desarrollo no ha sido siempre el mismo. Hace años, cuando el marketing era un torbellino de creatividad, el proceso estaba influido por un conjunto de circunstancias. Hoy día, la dinamicidad de las condiciones económicas, incluidas las expectativas de los accionistas y los factores de mercado, han hecho que estos directivos prefieran las extensiones de producto a la verdadera innovación.

Repasando algunas historias de éxito y volviendo a evaluar algunas prácticas comunes, se han descubierto mejoras concretas de procesos de fácil implementación que pueden ayudar al éxito de los nuevos productos.

Antes de las últimas convulsiones que han sacudido el mercado global, la mayoría de las empresas abogaban por el crecimiento y ansiaban "duplicar el negocio en un plazo de cinco años". Para conseguir este ambicioso objetivo, pusieron en marcha más estrategias e iniciativas que nunca. Ahora, el énfasis ya no está tanto en el crecimiento como en el mantenimiento de los ingresos actuales y en la reducción de gastos. A pesar de ello, ha aumentado el número de estrategias que se utilizan para conseguir estos objetivos.

Hubo una época, hace varias décadas, en la que los responsables del marketing eran las personas más creativas de la empresa.

Nuevos productos, nuevas campañas publicitarias y nuevas visiones de los consumidores eran el pan nuestro de cada día.

Hoy día, las cosas han cambiado, ya que la creatividad ha desaparecido y ya poco queda de ella en el desarrollo de nuevos productos.

Al utilizarse el mismo enfoque básico y rígido, se van a desarrollar y lanzar productos similares. La mayoría de ellos se parecerán y se producirá una falta de diferenciación. En la práctica, por tanto, este deseo de reducir el riesgo y la inversión directa no hace sino aumentar, al final, el riesgo de fracaso y de decepción. Y, a la inversa, si se implementan procesos basados solamente en un enfoque desestructurado, ¿cuáles son las posibilidades de que las ideas acaben viendo la luz? Una cosa son las buenas ideas y otra muy diferente es desarrollar un producto y lanzarlo con éxito y con la aceptación de los consumidores.

Ante esto y Tras años de extender las marcas con iniciativas lineales y repetitivas, es hora de retornar a las raíces creativas de las empresas. A continuación se exponen algunas formas sencillas de hacerlo:

- Al llevar a cabo una investigación de exploración, es importantes sentarse y pararse a escuchar y observar. Hay que hacerlo como si se estuviera ante una categoría nueva y olvidarse de los prejuicios y perspectivas anteriores.

-Al desarrollar proceso de ideación, la empresa debe contar con personas tanto de dentro como de fuera de ella. Si, por ejemplo, la empresa está creando una 
herramienta mecánica, debe incorporar a un quiropráctico para que ayude a entender mejor las implicaciones biofísicas de las necesidades del usuario junto con la tecnología y las ideas de la propia empresa.

- En el proceso de desarrollo y puesta a prueba de las ideas también se debe tomar en consideración a los consumidores en conjunto. La primera vez que se comparten ideas con los usuarios en potencia es necesario ofrecerles una breve descripción de lo que hace el producto en una o dos frases; continuación, hay que animarlos a hablar de la idea del producto en relación con su estilo de vida y de esta forma diseñar conceptos que cuenten una historia con la que los consumidores puedan identificarse.

Estos breves consejos ayudarán a encontrar un equilibrio entre las necesidades de la empresa y las del consumidor, hecho que redundará en un mayor retorno de la inversión en el nuevo producto.18 (Curewitz, Marekting e innovación el consumidor en el punto de mira.)

Método:

-El método utilizado en la investigación fue el Método científico, este método se caracteriza por ser: "1) sistemático significa que no puedo arbitrariamente eliminar pasos, sino que rigurosamente debo seguirlos; 2) metódico implica que se debe elegir un camino (método: camino hacia), sea, en este caso, una encuesta, una entrevista o una observación. 3) racional / reflexivo implica una reflexión por parte del investigador y tiene que ver con una ruptura con el sentido común. Hay que alejarse de la realidad construida por uno mismo, alejarse de las nociones, del saber inmediato. "19 (Morone)

-La técnica desarrollada fue la observación, "esta consiste en recibir conocimiento del mundo exterior a través de nuestros sentidos o el registro de información por medio de herramientas e instrumentos científicos. El proceso de observación científica consta de: 1) Observar algo y hacer preguntas acerca de un fenómeno natural (observación científica); 2) .Formular tu hipótesis; 3) Hacer predicciones sobre las consecuencias lógicas de la hipótesis; 4) Poner a prueba tus predicciones por medio de experimentos controlados, un experimento natural, un estudio observacional o un experimento de campo) 5) Crear tu conclusión sobre la base de los datos o la información reunida en el experimento".20 (Explorable.com, 2009)

- El instrumento escogido fue un estudio de caso exploratorio estos efectúan, normalmente," para aumentar el grado de familiaridad con fenómenos relativamente desconocidos, obtener información sobre la posibilidad de llevar a cabo una investigación más completa sobre un contexto particular de la vida real, investigar problemas del comportamiento humano que consideren cruciales los profesionales de determinada área, identificar conceptos o variables promisorias, establecer prioridades para investigaciones posteriores o sugerir afirmaciones (postulados) verificables"21 (Sampieri, 1991), en este caso aplica ya que es poco 
probable que alguien haya asociado la innovación y el marketing aplicados a una empresa como Barcelona Activa.

-Por último la herramienta aplicada fue una lista de chequeo, esta es una herramienta ampliamente utilizada en diagnósticos de cualquier área de índole gerencial y administrativa, estas listas presentaran una serie de variables que pueden ser consideradas como fortalezas o debilidades, estas se confrontaran para realizar un diagnóstico positivo, negativo o neutro; además se hace una breve explicación del por qué se considera cada variable una fortaleza y debilidad (en las conclusiones).

La primera lista de chequeo que se realiza a modo de diagnóstico incluye aspectos netamente de Marketing que serán resueltos en base a la situación de mercado de la empresa Barcelona Activa, esto permitirá demostrar en líneas generales si la empresa cumple con la utilización del primer pilar de la investigación, el marketing; la segunda lista incluye aspectos relacionados con lel segundo pilar: la innovación aplicada en las diferentes áreas de la compañía; estas dos listas permitirán verificar la relación de la innovación y el marketing no solo en la empresa si no en su servicio a los emprendedores, principal actividad de la compañía. Es de recalcar que ambos formatos base fueron facilitados por el profesor Héctor Horacio Murcia Cabra, en la catedra de Auditoria de séptimo semestre de la Universidad Santo Tomas.

\section{Resultados:}

1) Lista de chequeo de situación de marketing:

\begin{tabular}{|c|c|c|c|c|}
\hline \multicolumn{3}{|c|}{$\begin{array}{l}\text { Lista de chequeo de la situación de marketing Barcelona } \\
\text { activa. }\end{array}$} & Fortalezas & Debilidades \\
\hline \multirow[b]{4}{*}{ Oferta } & Tipo de p/s & $\begin{array}{l}\text { Barcelona activa tiene un factor } \\
\text { diferencial en sus servicios }\end{array}$ & $\mathrm{X}$ & \\
\hline & Cantidad & $\begin{array}{l}\text { cuenta con un buen portafolio de } \\
\text { productos }\end{array}$ & $x$ & \\
\hline & Calidad & $\begin{array}{l}\text { los clientes están satisfechos con } \\
\text { el servicio }\end{array}$ & $\mathrm{X}$ & \\
\hline & Cliente & $\begin{array}{l}\text { la empresa sabe lo que quiere su } \\
\text { cliente }\end{array}$ & $\mathrm{x}$ & \\
\hline \multirow[b]{2}{*}{ Demanda } & Segmentación & $\begin{array}{l}\text { Tiene claro cuál es su mercado } \\
\text { objetivo }\end{array}$ & $x$ & \\
\hline & Elasticidad & $\begin{array}{l}\text { Maneja un precio acorde a sus } \\
\text { servicios }\end{array}$ & $\mathrm{X}$ & \\
\hline
\end{tabular}




\begin{tabular}{|c|c|c|c|}
\hline & Competencia & $\begin{array}{l}\text { La empresa es fuerte frente a la } \\
\text { competencia. }\end{array}$ & $X$ \\
\hline \multirow[b]{2}{*}{ Precio } & Estacionalidad & $\begin{array}{l}\text { Barcelona activa recibe visitas de } \\
\text { clientes continuamente. }\end{array}$ & $X$ \\
\hline & Tendencia & $\begin{array}{l}\text { La empresa tiene un buen flujo de } \\
\text { clientes y de sponsors. }\end{array}$ & $X$ \\
\hline \multirow[b]{4}{*}{ Mercadeo } & Forma & $\begin{array}{c}\text { Barcelona activa presta sus } \\
\text { servicios de manera adecuada, }\end{array}$ & $X$ \\
\hline & Lugar & $\begin{array}{c}\text { Barcelona activa cuenta con una } \\
\text { infraestructura y espacio } \\
\text { adecuados. }\end{array}$ & $x$ \\
\hline & Tiempo & $\begin{array}{l}\text { Al momento de atender a un } \\
\text { cliente son eficientes en los } \\
\text { tiempos de atención y respuesta. }\end{array}$ & $X$ \\
\hline & Posesión & $\begin{array}{l}\text { Son buenos los canales de } \\
\text { difusión y posicionamiento que } \\
\text { manejan. }\end{array}$ & $X$ \\
\hline
\end{tabular}

\section{2) Lista de chequeo Innovación}

\begin{tabular}{|l|c|c|c|}
\hline \multicolumn{1}{|c|}{$\begin{array}{c}\text { Lista de chequeo Innovación en Barcelona } \\
\text { Activa }\end{array}$} & Fortaleza & $\begin{array}{c}\text { Información } \\
\text { insuficiente }\end{array}$ & Debilidad \\
\hline $\begin{array}{l}\text { 1. La empresa es innovadora en sus servicios } \\
\text { 2. Presenta Innovación en su procesos técnicos }\end{array}$ & $\mathrm{X}$ & \\
\hline $\begin{array}{l}\text { 3. Innova en la ampliación de su portafolio de } \\
\text { servicios. }\end{array}$ & $\mathrm{X}$ & & \\
\hline $\begin{array}{l}\text { 4. Ofrece asesorías para impulsar la innovación } \\
\text { 5. Apoya y fortalece la innovación entre sus } \\
\text { empleados. }\end{array}$ & $\mathrm{X}$ & & \\
\hline $\begin{array}{l}\text { 6. Innova para prestar un mejor servicio } \\
\text { 7. BA es innovadora frente a posibles } \\
\text { competidores }\end{array}$ & $\mathrm{X}$ & $\mathrm{X}$ & \\
\hline $\begin{array}{l}\text { 8. Fomenta la innovación en cada visita que } \\
\text { recibe. }\end{array}$ & & $\mathrm{X}$ & \\
\hline $\begin{array}{l}\text { 9. Es innovadora en convenios para realizar } \\
\text { alianzas estratégicas con organismos estatales } \\
\text { y privados. }\end{array}$ & $\mathrm{X}$ & & \\
\hline $\begin{array}{l}\text { 10. Apoya la innovación en otros lugares del } \\
\text { mundo. }\end{array}$ & $\mathrm{X}$ & & \\
\hline
\end{tabular}




\begin{tabular}{|c|c|}
\hline 11. BA innova en estrategias de marketing & $\mathrm{X}$ \\
\hline $\begin{array}{l}\text { 12. Utiliza medios y formas innovadoras de } \\
\text { publicidad difusión. }\end{array}$ & $\mathrm{X}$ \\
\hline
\end{tabular}

2)

\section{Conclusiones (Interpretación de resultados)}

De la investigación realizada se pueden extraer varias conclusiones:

-En primer lugar e interpretando los resultados se puede ver que la empresa Barcelona Activa es la empresa indicada para realizar este estudio, esto debido a que presenta una estructura de marketing optima y pone en práctica mecanismos innovadores:

De la primera lista de chequeo se extrae: primero que la empresa tiene un factor diferencial que es el de impulsar el emprendimiento y la innovación empresarial, además tiene un portafolio adecuado de servicios, desde el acompañamiento a emprendedores, a empresas, capacitaciones profesionales, promoción de sectores estratégicos, turismo y promoción de Barcelona y también una bolsa de empleo. Además BA tiene claro que su mercado objetivo: los emprendedores, la empresa brinda todo tipo de asesorías gratuitas y algunas con un costo muy accesible teniendo en cuenta la cantidad de su contenido

Al ser una empresa financiada en parte por el ayuntamiento de Barcelona no tiene una competencia directa, sin embargo está bien posicionada con organismos similares en España y Europa. La Compañía recibe cientos de visitantes a diario, de la ciudad y del exterior; es financiada en parte por el ayuntamiento de Barcelona pero también tiene alianzas estratégicas con organismos privados para mantener una adecuada liquidez y solvencia, según se pudo observar el servicio y disposición es óptima por parte de la compañía además de contar con una infraestructura tanto de panta como de equipo de primer nivel.

De la segunda lista de chequeo observamos que: Barcelona Activa es una empresa innovadora en términos generales, lastimosamente de algunos factores de la lista no se obtuvo suficiente información para hacer un diagnóstico, de los demás se extrae: BA es una empresa que innova constantemente en sus servicios, desde seminarios hasta grupos de investigación, consejeros vocacionales, tours por Barcelona, entre otros, la empresa y amplía su portafolio de servicios, además de la charla brindada se pudo observar que uno de los ejes de las políticas de apoyo al emprendimiento es que entre más innovadora sea la 
idea más posibilidades de apoyo de la empresa puede tener un ejemplo son las empresas enfocadas en apps que están un gran auge en la ciudad de Barcelona.

Se puede extraer además que la empresa al ser una de las pioneras en este modelo ha apoyado a varias ciudades del mundo (por ejemplo a Bogotá) a adoptar modelos para apoyar y desarrollar el emprendimiento local, la compañía también es capaz de innovar en la consecución de convenios privados y estatales, las únicas "debilidades" si se pueden llamar así tienen que ver con el hecho de que la empresa no es innovadora en términos de marketing publicitario, a pesar de que utiliza una agresiva publicidad en redes sociales no realiza innovación en esta área, sin embargo por el modelo de negocio manejado quizás sea lo más conveniente.

-En segundo lugar se puede concluir que la hipótesis: ¿es realmente el marketing un factor determinante en un proceso correcto de innovación empresarial? es verídica, esto teniendo en cuenta el marco referencial (artículos) y la visita a Barcelona Activa, basándonos principalmente en la actividad principal de la empresa: Fomentar el emprendimiento, para que el emprendimiento sea exitoso tiene que innovar de alguna u otra manera ya sea incrementalmente que "consiste en pequeñas modificaciones y mejoras que contribuyen, en un marco de continuidad, al aumento de la eficiencia o de la satisfacción del usuario o cliente de los productos y procesos" 22 (OCDE, 1997 ) o radicalmente que "se produce con productos y procesos nuevos, completamente diferentes a los que ya existen; son cambios revolucionarios en la tecnología y representan puntos de inflexión para las prácticas existentes”23 (Pedersen \& B. Dalum, 2004).

En consecuencia al tener que innovar en algún punto se tiene que involucrar el marketing, como se observó a lo largo de la investigación, en la justificación y en los artículos el marketing debe ser visto de forma integral, como herramienta que determina el éxito o fracaso de un producto, esto quiere decir que determina si una innovación es realizable o no. En Barcelona activa prestan un servicio muy completo de asesoría a los emprendedores, estudios de factibilidad, estudios demográficos, análisis de posibles mercados objetivo, asesorías contables y de logística, en pocas palabras se realiza un estudio de mercado para determinar si la idea de negocio es factible o no. Eso quiere decir que en cada proceso de innovación se deben tener en cuenta las variables que enmarcan al Marketing, como conclusión final podría afirmar que el marketing no solo es un factor fundamental para la innovación sino que son dos variables inherentes al éxito de un producto o servicio.

Lo anterior nos da para pensar en la posibilidad de instaurar en las universidades colombianas una catedra de la innovación enfocada al marketing: si ya hay cátedras de gerencia de mercadeo o de emprendimiento por que no crear una catedra de innovación en mercadotecnia, esto permitirá incentivar procesos de marketing innovadores desde las instituciones educativas; como segunda medida es necesario impulsar programas existentes como Bogotá Emprende con mayores recursos económicos que permitan generar una mayor difusión entre la sociedad 
colombiana, esto sumado a una inversión destinada a mejorar la infraestructura del programa ya que esta iniciativa no cuenta con una planta y equipo propia y es totalmente dependiente de la Cámara de Comercio de Bogotá, esto a pesar de manejar el mismo modelo de Barcelona Activa. Con estos recursos Bogotá emprende o el Parque del emprendimiento de Medellín puede servir como modelo para todas las ciudades del país y de esta forma generar una cultura de emprendimiento formal que aporte al crecimiento del país y no solo de unos pocos, así la sociedad colombiana podrá estar cada vez más cerca de sociedades innovadoras como la catalana.

Y a modo de reflexión final parece oportuna esta frase de Alejandro Schnarch Kirberg que creo enmarca todo lo aportado en esta investigación: "Definitivamente, creatividad sin innovación, es como un sueño; innovación sin creatividad, no puede existir; innovación sin marketing no tiene sentido. Creatividad, innovación y marketing en acción, son las claves del emprendimiento exitoso"24 (Kirberg)

\section{Bibliografía}

(A.M.A.), A. M. (s.f.). Dictionary of Marketing Terms. Obtenido de Marketing power: http://www.marketingpower.com/

Barcelona Activa. (s.f.). Quienes Somos. Obtenido de Barcelona Activa: http://www.barcelonactiva.cat/barcelonactiva/es/que-es-barcelona-activa/quienessomos/index.jsp

Curewitz, B. (s.f.). Marekting e innovación el consumidor en el punto de mira. Revista HarvardDeusto Marketing \& Ventas.

Curewitz, B. (s.f.). Marketing e innovación: el consumidor en el punto de mira. Revista HarvardDeusto Marketing \& Ventas.

Drucker, P. (1954). La práctica del management.

Explorable.com. (9 de Septiembre de 2009). Observación científica. Obtenido de Explorable.com: https://explorable.com/es/observacion-cientifica

KIRBERG, A. S. (2007). En Marketing Estratégico para Pymes . Global Educación.

KIRBERG, A. S. (2008). Creatividad Aplicada. Ecoe Ediciones.

Kirberg, A. S. (s.f.). Cretividad, innovacion y marketing. Obtenido de Avalon U tadeo: http://avalon.utadeo.edu.co/comunidades/egresados/portal/conferencia_julio21/creativi dad_innovacion_y_\%20marketing.pdf

Kotler, P. (2001). Dirección de Mercadotecnia 8va edicion. En P. Kotler. Hispanoamericana. 
Moreno, M. R. (2007). Cómo innovar en marketing . Revista MK. Marketing y Ventas para Directivos.

Morone, G. (s.f.). Métodos y técnicas de la investigación. Obtenido de Rua UNAM: http://www.rua.unam.mx/objeto/6852/metodos-y-tecnicas-de-la-investigacion-cientifica

OCDE. (1997) ). MANUAL DE OSLO. OSLO.

Pedersen, C., \& B. Dalum. (2004). Incremental versus radical: El caso del programa digital del norte de Dinamarca. En B. D. Pedersen.

Perez, J. F. (2009). Innovacion en Marketing. El éxito creativo de Atrapalo.com. Revista MK. Marketing y Ventas para Directivos.

Rivero, F. (2007). Innovacion en Marketing. MK Marketing + Ventas .

Sabater, S. M. (s.f.). Innovacion en Marketing. Obtenido de Club de marketng: www. clubdemarketing.com

Sampieri, R. H. (1991). Metodologia De La Investigacion-1. En R. H. Sampieri. México, D. F.: McGraw-Hill.

toledo, A. (s.f.). Marketing e Innovación: Los ingredientes perfectos para cocinar un negocio exitoso. Revista Puro Marketing, 12. 